https://doi.org/10.26512/gs.v10i3.26410

Revista Eletrônica Gestão \& Saúde ISSN: 1982 - 4785

Moreira FS; Maués LMF; Freitas ACA et al

Artigo Original

\title{
Bem-estar no Trabalho: Evidências na Indústria da Construção
}

\author{
Well-being at Work: Evidences in the Construction Industry
}

Bienestar en el trabajo: Evidencias en la industria de la construcción

Felipe de Sá Moreira ${ }^{1}$ Luiz Maurício Furtado Maués ${ }^{2}$ Ana
Carolina de Albuquerque Freitas $^{3}$ Claudionor Andrade Farias
Júnior $^{4}$

\section{Resumo}

Trabalhadores que atuam na indústria da construção são expostos a diversos fatores estressantes, e realizam tarefas exigentes em ambientes de trabalho perigosos, afetando a saúde, a segurança e o bem-estar. O objetivo desta pesquisa é identificar os principais aspectos que estão sendo relacionados ao bemestar no trabalho (BET) em profissionais do setor da construção. O método consiste na execução de uma Revisão Sistemática da Literatura (RSL) em 10 bases de dados internacionais. Um total de 93 publicações apontou 89 aspectos relacionados ao tema. Dentre os principais aspectos encontrados, $31 \%$ referem-se a medidas de BET e estresse, $19,8 \%$ a saúde física, $14,5 \%$ a segurança, $6,1 \%$

\footnotetext{
${ }^{1}$ Engenheiro Civil. Mestrado em engenharia civil. Discente de
doutorado / Universidade Federal do Pará E-mail:

${ }^{1}$ Engenheiro Civil. Mestrado em engenharia civil. Discente de
doutorado / Universidade Federal do Pará E-mail: samoreira@ufpa.br / https://orcid.org/0000-0002-7169-0534
${ }^{2}$ Engenheiro Civil. Doutorado em engenharia civil. Professo
/ Universidade Federal do Pará. E-mail: maues@ufpa.b

samoreira@ ufpa.br / https://orcid.org/0000-0002-7169-0534
${ }^{2}$ Engenheiro Civil. Doutorado em engenharia civil. Professor
/ Universidade Federal do Pará. E-mail: maues@ ufpa.br samoreira@ufpa.br / https://orcid.org/0000-0002-7169-0534
${ }^{2}$ Engenheiro Civil. Doutorado em engenharia civil. Professor
/ Universidade Federal do Pará. E-mail: maues@ufpa.br https://orcid.org/0000-0002-1762-8617
}

a saúde mental e 4,4\% a aspectos gerais. Os achados desta pesquisa refletem razoavelmente a singularidade dos profissionais da indústria da construção e ratificam a necessidade de uma abordagem convergente a respeito do tema, que, certamente, poderia proporcionar práticas mais eficientes e objetivas de fomento ao bem-estar. Descritores: saúde, indústria da construção, revisão sistemática.

\begin{abstract}
Workers in the construction industry are exposed to various stressors, and perform demanding tasks in hazardous work environments and affect health, safety and well-being. The objective of this research is to

\footnotetext{
${ }^{3}$ Acadêmica de Engenharia Civil. Acadêmico de engenharia civil / Universidade Federal do Pará. E-mail: carol.albuquerquef@gmail.com https://orcid.org/0000-0002$\underline{1188-1849}$

${ }^{4}$ Engenheiro Civil. Mestrado em engenharia civil. Discente de doutorado / Universidade Federal do Pará. E-mail: claudionorfj@gmail.com https://orcid.org/0000-0001-8582$\underline{6191}$
} 
point out the main aspects that are being related to the well-being at work (WBW) in professionals of the construction sector. The method consists in the execution of a Systematic Review of Literature (SRL) in 10 international databases. 93 publications pointed out 89 aspects related to the theme. Among the main aspects found, $31 \%$ refer to WBW and stress, $19.8 \%$ to physical health, $14.5 \%$ to safety, $6.1 \%$ to mental health and $4.4 \%$ to general aspects. The findings of this research reasonably reflect the uniqueness of the construction industry professionals and ratify the need for a convergent approach to the subject, which could certainly provide more efficient and objective practices for fostering well-being.

Key-words: health, construction industry, systematic review.

\section{Resumen}

Los trabajadores de la industria de la construcción están expuestos a una variedad de factores estresantes y realizan tareas exigentes en entornos de trabajo peligrosos, que afectan la salud, la seguridad y el bienestar. El objetivo de esta investigación es identificar los principales aspectos que se relacionan con el bienestar en el trabajo (BET) en los profesionales del sector de la construcción. El método consiste en realizar una Revisión Sistemática de la Literatura (RSL) en 10 bases de datos internacionales. Un total de 93 publicaciones mencionaron 89 aspectos relacionados con el tema. Entre los principales Rev. Gestão \& Saúde (Brasília) Vol. 11, n. 01, Jan. 2020. aspectos encontrados, $31 \%$ se refieren a BET y medidas de estrés, $19.8 \%$ a salud física, $14.5 \%$ a seguridad, $6.1 \%$ a salud mental y $4.4 \%$ a aspectos generales. Los resultados de esta investigación reflejan razonablemente la singularidad de los profesionales en la industria de la construcción y ratifican la necesidad de un enfoque convergente sobre el tema, que sin duda podría proporcionar prácticas más eficientes y objetivas de promoción del bienestar.

Descriptores: salud, industria de la construcción, revisión sistemática.

\section{Introdução}

O termo bem-estar é definido sob luz de duas perspectivas distintas(1,2): a) a hedonista, na qual o bem-estar subjetivo se fundamenta, e b) a eudaimonista, a qual baseia-se no bemestar psicológico. A definiç̧ão do bem-estar hedonista centra-se no estudo das emoções positivas e negativas assim como na satisfação de vida(1,3-7). Já a perspectiva eudaimonista, compreende o bem-estar como um conjunto de 6 fatores: autonomia, crescimento pessoal, auto aceitação, propósito de vida, domínio e relação positiva(1,4,7-10).

Com a evolução destas perspectivas ao longo do tempo, o bem-estar passou de uma percepção popular para uma medida de interesse global, o qual, atualmente, é incluído em análises de desenvolvimento humano(11), além de ser um tema de prioridade em governos, organizações internacionais, 
empresas de saúde e instituições de pesquisa(12). Estudos voltados às classes específicas de trabalhadores são desenvolvidos ao longo do tempo e proporcionam um olhar singular deste tema para cada labor. Nesta perspectiva, esta pesquisa tem o objetivo de apontar os principais aspectos que estão sendo relacionados ao BET em profissionais do setor da construção, através de uma pesquisa exploratória utilizando uma revisão sistemática da literatura (RSL). Para atender tal objetivo, utilizaram-se 10 bases de publicações reconhecidas internacionalmente.

\section{Referencial teórico}

O construto bem-estar pode ser estudado sem um determinado contexto (como o bem-estar da vida como um todo) ou associado a uma única área da vida(13). Em geral, as principais pesquisas na área referemse ao bem-estar geral, desvinculado de contexto(14). Ao longo do tempo, pesquisas foram expandidas e direcionadas para diversos âmbitos, sendo um deles o ambiente de trabalho(15), sobre o qual trata esta pesquisa.

$\mathrm{Na}$ academia, fica evidente a importância do tema pelo número e evolução de publicações que fazem referência ao construto. Em uma pesquisa na base científica Scopus com os termos "happiness" ou "wellbeing" vinculados ao termo "work" foram obtidas 20.116 publicações. Até 1960 registrou-se apenas 43 publicações, entretanto no último período (2011 a 2018), foram encontradas 11.402 publicações relacionadas Rev. Gestão \& Saúde (Brasília) Vol. 11, n. 01, Jan. 2020. ao tema, demonstrando o crescente interesse científico no assunto.

Outro aspecto que fundamenta a relevância do tema é sua relação direta com a qualidade da vida humana, que por si só já justificaria pesquisas na área. Além disso, os trabalhadores, que são o objeto de estudo, passam boa parte de suas vidas dedicando-se ao labor(16) e representam parte expressiva da população global, contribuindo significativamente para o valor econômico e social da sociedade(17).

Apesar de não ser recente, existe uma necessidade de convergência deste tema na literatura, já que as abordagens atuais não permitem uma visão plena e integrada do bemestar no trabalho (BET)(18). Estudos que desenvolvem tal temática, tratam de diferentes conceitos como estresse, exaustão emocional(19), relacionamento interpessoal, ambiente, natureza da tarefa(20), engajamento e burnout(21), por exemplo. Entretanto, termos como satisfação, envolvimento, comprometimento, engajamento, estado de fluxo e motivação são os principais encontrados na academia ligados ao construto(18), sendo alguns deles frequentemente apresentados como sinônimos de bem-estar. Fisher(15) e Siqueira et. al(22) fundamentam como medidas de BET a satisfação (julgamento cognitivo sobre aspectos presentes no ambiente de trabalho), o engajamento (envolvimento afetivo e cognitivo de desfrute do próprio trabalho) e comprometimento organizacional afetivo 
(sentimentos e apegos dirigidos à organização que o emprega).

Quanto à indústria da construção, esta se mostra particularmente insalubre já que sua taxa de doenças relacionadas ao trabalho é uma das mais altas em todos os grupos ocupacionais(23). Tal fato, torna um desafio o desenvolvimento efetivo da saúde ocupacional neste ramo(24).

Os profissionais que atuam neste nicho são expostos a diversos fatores estressantes(25,26), que têm impactos negativos em seu bem-estar mental, os quais refletem no desempenho individual e organizacional(27), incluindo: longas horas e elevada carga de trabalho, conflito de papéis, insegurança (tanto física, quanto ao emprego), insatisfação, pressões elevadas e conflito entre trabalho e família(28,29). Para Jebelli et al.(30), tarefas psicologicamente e fisiologicamente exigentes, realizadas em ambientes de trabalho perigosos, fazem da atividade de construção uma das ocupações mais estressantes, o que afeta a produtividade, a segurança, o bem-estar e a qualidade do trabalho.

Outras características inerentes ao setor da construção corroboram ainda mais com a sua singularidade e risco: possui caráter itinerante, o que pode incentivar estilos de vida menos saudáveis(31); seus trabalhadores são pouco qualificados(32,33) e suscetíveis a ingestão de álcool e fumo(34,35); é cíclico devido à suscetibilidade aos aspectos econômicos(36); é dominado por homens, Rev. Gestão \& Saúde (Brasília) Vol. 11, n. 01, Jan. 2020. apresentando estigmas e culturas preponderantemente masculinas $(29,37)$; possui elevado índice de absenteísmo e rotatividade $(38,39)$; por outro lado um baixo índice de produtividade $(32,40,41)$; bem como de comprometimento(42).

Apesar de existirem iniciativas para estimular o aumento dos programas de bemestar no local de trabalho na indústria da construção, poucas intervenções abordam a temática de uma forma completa ou global(43). Estudos concentrados em fatores psicossociais têm focado principalmente nos aspectos negativos da saúde, assim como em resultados como acidentes ocupacionais(44). Por isso, é preciso realizar pesquisas para determinar a extensão do problema, ou seja, um estudo que possa avaliar o bem-estar através do uso de ferramentas de diagnóstico bem estabelecidas, uma vez que não há estudos empirícos com tal abordagem(28).

Encontram-se diversas pesquisas de RSL que abordam o BET de forma geral, assim como em contextos específicos, entretanto, não foi identificado nenhum exclusivamente para o contexto da indústria da construção. Hirschle e Gondim(45), por exemplo, exploraram as relações entre estresse e BET. Estas autoras apresentaram evidências empíricas de variáveis pessoais e ambientais protetoras dos efeitos negativos do estresse no bem-estar. Já Santos e Ceballos(46) buscaram as principais publicações que abordam o BET e apontaram a preocupação dos estudos brasileiros em conceituar o tema; a diversidade de 
instrumentos/medidas utilizados; e os aspectos positivos e negativos que interferem no BET. Haggman-Laitila e Romppanen $(47,48)$ revisaram a literatura com o objetivo de sintetizar o conhecimento atual da pesquisa sobre intervenções que buscam melhorar o BET em enfermeiros. Assim, a relevância a respeito do tema aliada a especificidade do setor da construção frente às outras indústrias, expõem uma importante lacuna do conhecimento a ser explorada.

\section{Método}

A presente RSL foi desenvolvida no período de novembro de 2018 a abril de 2019 e registrada no banco de registro internacional de revisões sistemáticas PROSPERO, sendo vinculada à seguinte numeração de cadastro/busca: CRD42019122233. Neste banco de registro não foram encontradas RSL abordando a temática deste estudo. Para a execução desta pesquisa, foi utilizado o software START (State of the Art through Systematic Review). O método aplicado inicia com a fase do estudo exploratório, no qual buscou-se, além de uma melhor familiarização com o tema, a determinação das condições de contorno da RSL.

As bases de dados foram definidas através do cruzamento de três referências: $\left(1^{\mathrm{a}}\right)$ 27 artigos de RSL obtidos de forma exploratória voltados para o BET; $\left(2^{\mathrm{a}}\right)$ os autores Morandi e Camargo(49); (3ª $)$ o próprio conhecimento empírico dos autores desta pesquisa. Foram selecionadas as bases mais Rev. Gestão \& Saúde (Brasília) Vol. 11, n. 01, Jan. 2020. utilizadas nestes artigos com base no princípio de Pareto. Outro aspecto importante é a utilização de bases correlatas à engenharia civil, que fornece a perspectiva dos profissionais e pesquisadores que atuam diretamente na área. Assim, 10 bases foram escolhidas para esta RSL: EBSCO, ProQuest, PubMed (Natinal Library of Medicine), Scopus, Web of Science, Cochrane Library, Scielo (Scientific Electronic Library Online), Emerald, PsycINFO (American Psychological Association) e Engineering Village.

As palavras-chaves utilizadas foram concentradas metodologicamente em 2 grupos: um referenciado ao bem-estar ("well-beng", "wellbeing" e "well being"); e outro à indústria da construção ("civil construction", "construction industry", "construction sector", "construction sites", "construction workers"). Não foram utilizados termos isolados como "construction" ou "building" uma vez que retomaram um número expressivo de artigos irrelevantes ao tema desta pesquisa. A string de pesquisa foi elaborada utilizando o operador boleano "AND" entre os grupos, assim como o operador " $O R$ ” entre os sinônimos de cada grupo. Não se limitou idioma nem período de publicação, entretanto os estudos devem possuir ao menos o título ou resumo na língua inglesa, uma vez que os termos da pesquisa se encontram nesta língua. Além disso, as buscas foram feitas nos campos título, resumo e palavras-chaves.

A Figura 1 apresenta os passos seguidos para a operacionalização desta RSL. 
A princípio, foi obtido um total de 988 artigos nas buscas nas bases de dados, e retirados 575 duplicados, assim como 6 erros de importação, resultando em 407 artigos. Na fase seguinte, a de seleção, os títulos e resumos são lidos com $\mathrm{o}$ intuito de verificar a aderência ao propósito da pesquisa. Para isto, os seguintes critérios de exclusão foram estipulados: (a) configurar-se como editoriais, resumos ou chamadas de congressos, de conferências ou de periódicos, (b) caracterizar-se como artigos de revisão de literatura, (c) não possuir ou tratar explicitamente de aspecto relacionado ao BET, (d) não possuir exclusivamente em sua amostra ou objeto de pesquisa profissionais atrelados diretamente a atividade de construção. Nesta etapa foram obtidos 166 artigos e rejeitados 241.

$\mathrm{Na} 3^{\mathrm{a}}$ etapa, a de extração, a leitura Figura 1 - Etapas da pesquisa detalhada dos artigos é feita na íntegra e os mesmos critérios são aplicados. Tal procedimento torna-se necessário, uma vez que não é possível em todos os casos identificar as características de exclusão somente com a leitura do título e resumo dos artigos. Fora isto, foram inclusos mais dois critérios de exclusão: possuir 2 páginas ou menos; e trabalhos que não estão disponíveis para os autores, por ser um trabalho antigo, por exemplo. No final, obtiveram-se 92 trabalhos, nos quais foram feitas as extrações/sínteses das suas principais informações: ano de publicação, tipo (artigo de periódico, de conferência, tese ou dissertação), autores, país de estudo, tipo e tamanho da amostra, objetivo, resultados da pesquisa e aspectos relacionados ao bem-estar. 


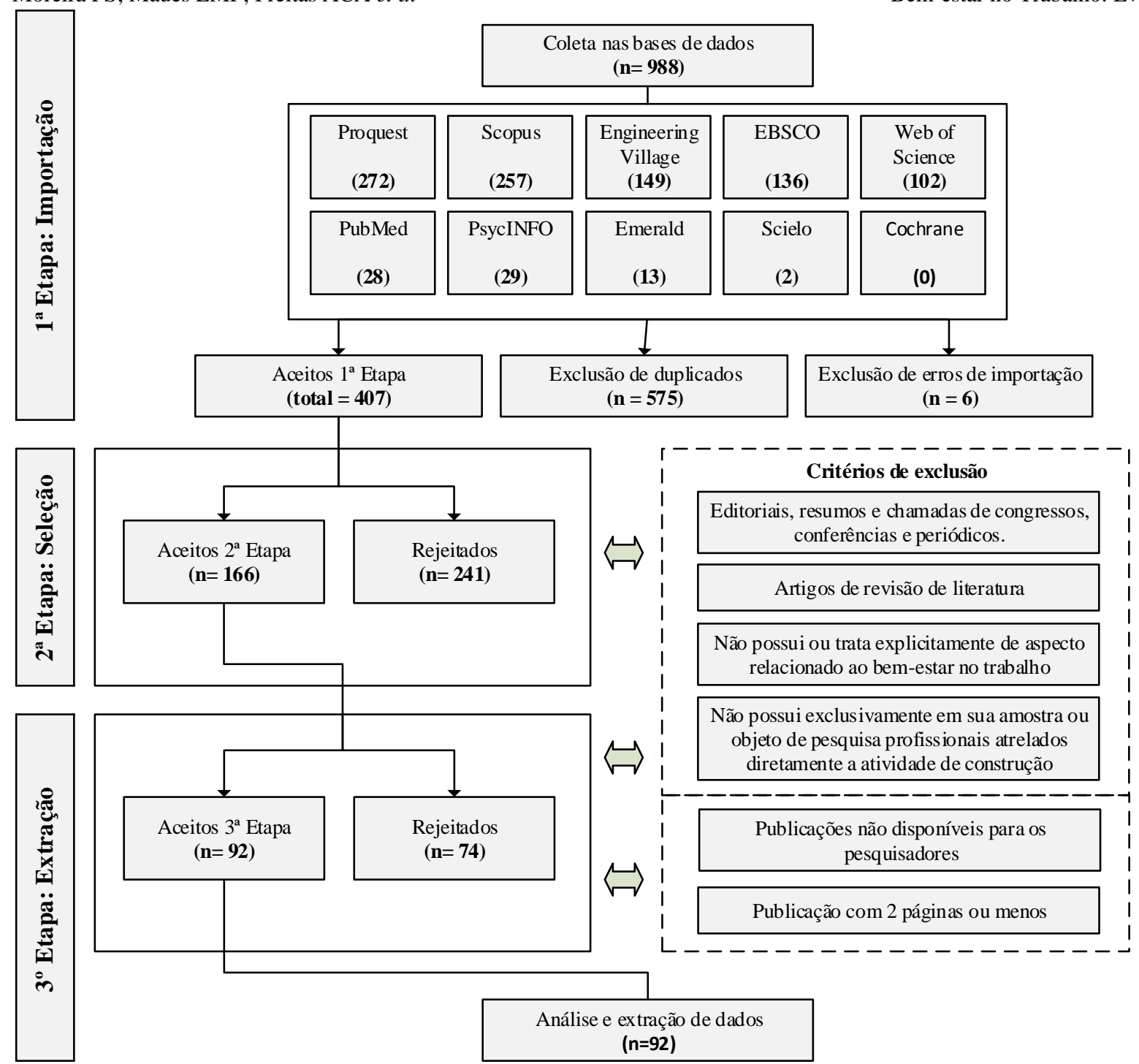

Fonte: autoria própria.

\section{Resultados}

Os principais autores encontrados nas 92 publicações, assim como os principais países em que estes estudos foram desenvolvidos estão apresentados no Quadro 1. Estes países são: Estados Unidos, no qual foi encontrado o maior número de publicações, um total de 21 estudos (22,83\%); na Austrália,
18 (19,56\%); no Reino Unido, 14 (15,22\%); África do Sul, 9 (9,78\%); Espanha 5 (5,43\%), China, 5 (5,43\%); e Canadá, 3 (3,26\%). Outros apresentaram 2 publicações ou menos, fato que mostra a baixa representatividade destas nações em relação ao tema desta pesquisa. São eles: Brasil, Índia, Inglaterra, Singapura (com 2 publicações cada), Coréia, Egito, Filipinas, Hong Kong, Malásia, Nigéria, Qatar, Siri Lanka e Suécia (com 1 publicação cada).

Quadro 1 - Principais autores por país 


\begin{tabular}{|c|c|}
\hline Países & Autores \\
\hline Estados Unidos & $\begin{array}{l}\text { Abraham(50); Buck(51); Choi(52); Gatti et al.(53); Hinze et al.(54); Albert et } \\
\text { al.(55); Bodner et al.(56); Costa(57); Gatti et al.(58); Kaufman et al.(59); Arias et } \\
\text { al.(60); Dailey(61); Lee et al.(62); Gatti et al.(63); Anger et al.(43); Curtis et } \\
\text { al.(64); Jebelli et al.(65); Jebelli et al.(30); Peters et al.(66); Thomas(67); Tosti(68) }\end{array}$ \\
\hline Austrália & $\begin{array}{l}\text { Lingard e Francis(69); Love et al.(70); Turner e Lingard(71); Townsend et al.(36); } \\
\text { Du Plessis et al.(29); Milner et al.(72); Zacher et al.(73); Milner et al.(74); Turner } \\
\text { e Lingard(75); Turner e Mariani(76); Loudoun e Townsend(77); Pidd et al.(78); } \\
\text { Sunindijo e Kamardeen(27); King et al.(79); Kurtzer et al.(80); Langdon e } \\
\text { Sawang(81); Powell et al.(37); Zafar e Chileshe(34) }\end{array}$ \\
\hline Reino Unido & $\begin{array}{l}\text { Sutherland e Davidson(82); Love e Edwards(83); Sang et al.(28); Sang et al.(84); } \\
\text { Sang et al.(85); Tyers et al.(86); Sherratt(87); Aboagye-Nimo et al.(88); Eaves et } \\
\text { al.(89); Gibb et al.(24); Eaves et al.(90); Valero et al.(91); Cole(92); Cole(93) }\end{array}$ \\
\hline África do Sul & $\begin{array}{l}\text { Deacon et al.(23); Smallwood e Haupt(94); Mostert et al.(21); Bowen et al.(26); } \\
\text { Bowen et al.(95); Bowen et al.(96); Bernstein e Trimm(97); Coetzer et al.(98); } \\
\text { Obiozo e Smallwood(99) }\end{array}$ \\
\hline Espanha & $\begin{array}{l}\text { Meliá e Becerril(100); Fernández et al.(101); Navarro-Astor(102); López-Araújo } \\
\text { e Segovia(103); Lorente et al.(44) }\end{array}$ \\
\hline China & Chan et al.(104); Yi e Chan(105); Yi et al.(106); Kong et al.(107); Yuan et al.(108) \\
\hline Canadá & McCabe et al.(109); Alwasel et al.(110); Chen et al.(111) \\
\hline
\end{tabular}

Fonte: autoria própria.

De forma geral, percebe-se também que a frequência de publicações destes estudos vem aumentando periodicamente. Até 2005 foram encontradas 5 publicações; de 2006 a 2010, 12; de 2011 a 2015, 36; e de 2016 até a presente pesquisa, 39. Quanto ao tipo de publicação, encontraram-se preponderantemente artigos de periódicos (69), sendo o restante dos estudos classificados em artigos de conferência (12), de revista (5), de congresso (2), dissertação de mestrado (2), capítulo de livro (1) e tese de doutorado (1). De fato, as bases de busca selecionadas para esta pesquisa possuem, em sua maioria, elevado número de artigos de periódicos, o que justifica tal resultado. Em relação às populações estudadas, 73 publicações possuíam em sua amostra operários de obra, 31 

suporte e apoio social (com 17 publicações) e presença de um tipo de profissional em uma publicação, não exclui a abordagem de outro, ou seja, há trabalhos que pesquisaram concomitantemente todos os citados, por exemplo.

Foram encontrados 89 aspectos relacionados ao tema desta pesquisa, dentre os quais: 3 referem-se a aspectos gerais ou globais; 32 relacionam-se a fatores de cada indivíduo; e 54 são aspectos organizacionais. Tal classificação foi baseada na visão de Sender e Fleck(18), as quais afirmam que a origem do BET pode se situar no indivíduo ou na organização, uma vez que as características de cada pessoa assim como as iniciativas da organização podem influenciar o contexto do trabalho. Além disto, é relevante categorizar os aspectos em mentais, físicos e ambientais, conforme mostrado no Quadro 2.

Dentre os principais aspectos obtidos encontram-se (a) o estresse e (b) a segurança, (d) distúrbios músculo esqueléticos (lesões e dores) e (e) equilíbrio/conflito entre trabalho e família, com 16 publicações cada. Percebe-se significativa diversidade observando todos os aspectos encontrados nesta pesquisa. Logo, com objetivo de se realizar uma análise consistente e lógica, apresenta-se o Quadro 3, o qual mostra os aspectos mais abordados nas publicações, agrupados por afinidade. Este foi obtido através de dois passos: $\left(1^{\circ}\right)$ inicialmente um diagrama de Pareto foi traçado com base no número de publicações que abordam os aspectos, sendo apresentados a somatória representativa acumulada de $80 \%$; $\left(2^{\circ}\right)$ posteriormente estes aspectos foram agrupados por afinidade. Os grupos encontrados foram: (a) medidas de BET, estresse e seus estressores; (b) saúde física global; (c) segurança; (d) saúde mental; (e) geral; e (f) outros. 
Quadro 2 - Aspectos mapeados relacionados ao BET

\begin{tabular}{|c|c|c|}
\hline Foco & Aspectos & \\
\hline \multirow{6}{*}{ Organização } & \multicolumn{2}{|l|}{ Mentais } \\
\hline & \multicolumn{2}{|c|}{$\begin{array}{l}\text { (19) Estresse; (17) Suporte e apoio social; (16) Equilíbrio/conflito entre } \\
\text { trabalho/vida; (10) Controle do trabalho; (9) Satisfação no trabalho; (6) Pressão } \\
\text { no trabalho; (5) Assédio moral; (5) Liderança; (4) Clima de segurança; (4) } \\
\text { Engajamento; (4) Intenções de rotatividade; (3) Desempenho auto avaliado; (3) } \\
\text { Responsabilidade pela segurança do outro; (2) Assédio sexual; (2) Autonomia; } \\
\text { (2) Conflitos de papéis; (2) Conflito interpessoal; (2) Discriminação; (2) } \\
\text { Percepção de risco/segurança; (2) Recursos pessoais; (2) Relações positivas de } \\
\text { trabalho; (1) Compromisso da gestão com a segurança; (1) Conhecimento de } \\
\text { segurança; (1) Conscienciosidade; (1) Envolvimento no trabalho; (1) Imersão no } \\
\text { trabalho; (1) Incerteza financeira; (1) Intimidação no trabalho; (1) Justiça } \\
\text { organizacional; (1) Meditação; (1) Motivação de segurança; (1) Percepção do } \\
\text { compromisso de segurança do supervisor/colega; (1) Qualidade percebida do } \\
\text { trabalho; (1) Rede social e capital; (1) Relacionamento supervisor x subordinado; } \\
\text { (1) Sindicalização; (1) Tokenismo. }\end{array}$} \\
\hline & Físicos & Físicos e Mentais \\
\hline & $\begin{array}{l}\text { (3) Sintomas físicos de estresse; } \\
\text { (2) Limitações funcionais; (1) } \\
\text { Demanda física/gasto de energia }\end{array}$ & $\begin{array}{l}\text { (14) Demanda do trabalho; (5) Burnout; (4) } \\
\text { Recursos de trabalho; (2) Capacidade de } \\
\text { trabalho; (2) Horas excessivas de trabalho; } \\
\text { (1) Limitações no trabalho; (1) Sobrecarga de } \\
\text { papéis; (1) Sobrecompensação. }\end{array}$ \\
\hline & \multicolumn{2}{|l|}{ Ambientais } \\
\hline & \multicolumn{2}{|c|}{$\begin{array}{l}\text { (19) Segurança; (14) Exposições físicas; (11) Aspectos ergonômicos do trabalho; } \\
\text { (2) Layout do canteiro; (1) Condições de trabalho; (1) Design do trabalho. }\end{array}$} \\
\hline \multirow[b]{2}{*}{ Indivíduo } & Mentais & Físicos \\
\hline & $\begin{array}{l}\text { (13) Bem-estar mental / } \\
\text { sofrimento mental; (7) Bem-estar } \\
\text { psicológico; (4) Suicídio; (2) } \\
\text { Autoestima; (2) Distúrbio } \\
\text { emocional/mental; } \\
\text { Personalidade negativa } \\
\text { personalidade; (1) Abertura a }\end{array}$ & 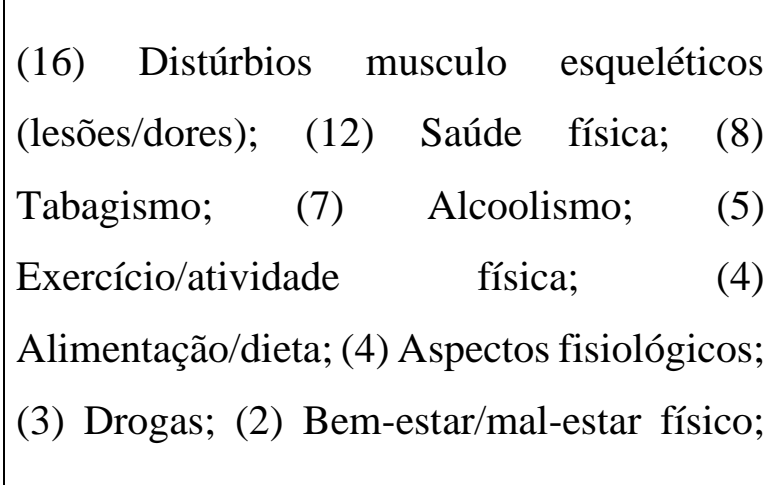 \\
\hline
\end{tabular}


Revista Eletrônica Gestão \& Saúde ISSN: 1982 - 4785

Moreira FS; Maués LMF; Freitas ACA et al Bem-estar no Trabalho: Evidências

Moreira FS; Maués LMF; Freitas ACA et al Bem-estar no Trabalho: Evidências...

mudanças; (1) Autenticidade; (1)|(2) Fadiga/exaustão; (2) Insônia; (2) Privação

Bem-estar social; (1) Emoções de sono; (1) Qualidade do sono.

positivas (1) Exaustão emocional;

(1) Fatalismo; (1) Felicidade; (1)

Habilidade de comunicação; (1)

Inteligência emocional; (1)

Isolamento/solidão;

(1)

Resiliência; (1) Temperamento

pessoal; (1) Virtude cívica

extrafuncional;

\section{Gerais/Globais}

(8) Importância do BET; (7) Avaliação/Implantação de programas de saúde; (1) Qualidade de vida dos profissionais.

Fonte: autoria própria. 
Quadro 3 - Principais aspectos

\begin{tabular}{|c|c|}
\hline Aspectos & Grupo \\
\hline $\begin{array}{l}(5,46 \%) \text { Estresse; }(4,89 \%) \text { Suporte e apoio social; }(4,6 \%) \\
\text { Equilíbrio/conflito entre trabalho e família; }(4,02 \%) \text { Demanda do } \\
\text { trabalho; }(2,87 \%) \text { Controle do trabalho; }(2,59 \%) \text { Satisfação no trabalho; } \\
(1,72 \%) \text { Pressão no trabalho; }(1,44 \%) \text { Assédio moral; }(1,44 \%) \text { Burnout; } \\
(1,15 \%) \text { Engajamento; }(1,15 \%) \text { Recursos de trabalho }\end{array}$ & $\begin{array}{l}\text { Medidas de } \\
\text { BET, } \\
\text { estresse e } \\
\text { estressores } \\
(31,33 \%)\end{array}$ \\
\hline $\begin{array}{l}\text { (4,6\%) Distúrbios musculo esqueléticos (lesões/dores); }(3,45 \%) \text { Saúde } \\
\text { física; } \quad(2,3 \%) \quad \text { Tabagismo; } \quad(2,01 \%) \quad \text { Alcoolismo; }(1,44 \%) \\
\text { Exercício/atividade física; } \quad(1,15 \%) \text { Alimentação/dieta; }(1,15 \%) \\
\text { Aspectos fisiológicos; }(0,86 \%) \text { Drogas; }(0,86 \%) \text { Sintomas físicos de } \\
\text { estresse }\end{array}$ & $\begin{array}{l}\text { Saúde física } \\
\text { global } \\
(17,82 \%)\end{array}$ \\
\hline $\begin{array}{l}(5,46 \%) \text { Segurança; }(4,02 \%) \text { Exposições físicas; }(3,16 \%) \text { Aspectos } \\
\text { ergonômicos do trabalho; }(1,15 \%) \text { Clima de segurança; }(0,86 \%) \\
\text { Responsabilidade pela segurança do outro }\end{array}$ & $\begin{array}{l}\text { Segurança } \\
(14,65 \%)\end{array}$ \\
\hline $\begin{array}{l}(3,74 \%) \text { Bem-estar mental / sofrimento mental; (2,01\%) Bem-estar } \\
\text { psicológico }\end{array}$ & $\begin{array}{l}\text { Saúde mental } \\
(5,75 \%)\end{array}$ \\
\hline $\begin{array}{l}(2,01 \%) \text { Programa de saúde total; }(2,3 \%) \text { Gerais sobre bem-estar } \\
\text { (importância) }\end{array}$ & $\begin{array}{l}\text { Geral } \\
(4,31 \%)\end{array}$ \\
\hline $\begin{array}{l}(1,44 \%) \text { Liderança; }(1,15 \%) \text { Suicídio; }(1,15 \%) \text { Intenções de } \\
\text { rotatividade; } \\
(0,86 \%) \text { Desempenho auto avaliado }\end{array}$ & $\begin{array}{l}\text { Outros } \\
(4,6 \%)\end{array}$ \\
\hline
\end{tabular}

Fonte: autoria própria.

\section{Discussão}

A heterogeneidade dos conceitos e abordagens a respeito do bem-estar livre de contexto, assim como do próprio BET, são percebidos de forma nítida quando analisado os resultados desta pesquisa, fato que que ratifica a visão de Sender e Fleck(18). Isto se explica através dos 89 aspectos encontrados (ver Quadro 2), os quais não apresentam clareza de convergência entre si de forma concisa. Também não se pode concluir que a elevada frequência de citações ou estudos a respeito de certos aspectos relacionados a medida de BET (ver Quadro 3) indiquem esta convergência. Mas tal fato pode indicar um caminho a ser traçado para obtê-la.

Recentemente, estas mesmas autoras realizaram um estudo de mapeamento e análise dos questionários que medem a felicidade no trabalho, o qual foi tomado como um conceito "guarda-chuva". Os principais construtos 
academicamente vinculados a este conceito macro foram satisfação, bem-estar, comprometimento, engajamento, motivação, envolvimento e estado de fluxo(18). Dentre estes, apenas a satisfação e o engajamento foram mapeados na atual RSL, o que pode indicar um distanciamento ou escassez destes estudos no setor da construção.

Em consonância, Santos e Cerballos(46) constataram significativa variedade de instrumento de avaliação do BET, fato que mostra a falta de um indicador comum entre os pesquisadores. Dentre os resultados destes pesquisadores, encontram-se escalas e medidas negativas, como estresse ocupacional, exaustão emocional, pressão no trabalho e sintomas de depressão, bem como engajamento, comprometimento, autoestima e otimismo.

Fora a diversidade, a maneira e a extensão com que os fatores que afetam potencialmente os resultados de bem-estar interagem, parece não ter sido documentados de maneira sistemática, apresentando-se como uma oportunidade para mais pesquisas(29). A complexidade, abrangência e qualidade multifacetada do bem-estar podem justificar tal problema, entretanto não exclui a necessidade de uma abordagem convergente a respeito do tema, que, certamente, poderia proporcionar práticas mais eficientes e objetivas de fomento ao bem-estar.

Dentre as iniciativas presentes nos canteiros de obra, encontram-se os Programas Rev. Gestão \& Saúde (Brasília) Vol. 11, n. 01, Jan. 2020. de Saúde Total (Total Worker Health), que se configuram como intervenções que visam estimular de forma global o bem-estar aos trabalhadores. Eles abordam, em muitos casos, somente aspectos relacionados a saúde física, mas sua concepção mais abrangente engloba também a saúde mental, assim como o próprio ambiente físico. Nacionalmente, pouco se percebe programas com esta abordagem global no contexto da indústria da construção. $\mathrm{Na}$ literatura encontrada isto também é refletido: em 8 publicações que trataram sobre o tema, 3 foram sobre programas utilizados dos Estados Unidos, 3 no Reino Unido e 2 na Austrália.

De fato, um indicador preocupante é a concentração de publicações relacionadas ao assunto em países desenvolvidos: se computarmos somente as publicações destes mesmos 3 países citados anteriormente, chegamos a 53 estudos do total de publicações retomadas neste trabalho, ou seja, 57\%. Tal dado pode indicar que países em desenvolvimento e subdesenvolvidos pouco estudam esta temática, mesmo com tal relevância. Uma possível explicação seria o baixo grau evolutivo de bem-estar dos trabalhadores em que estes países se encontram, preocupando-se ainda com aspectos básicos do trabalho.

Quando se indica a necessidade de análises globais, ou seja, com uma visão holística do trabalho e do trabalhador que interferem nele e na própria organização, é fundamental considerar as características 
específicas de cada função. Assim, características laborais singulares de cada atividade podem modificar os aspectos analisados relativos ao bem-estar do trabalho, permitindo (com a modificação ou criação de novos métodos de medição) não só a obtenção de melhores resultados, mas também modelos mais aderentes às funções de cada trabalhador.

Tal especificidade foi tratada por Goldenhar et al.(112), os quais relacionaram os estressores e seus efeitos em trabalhadores do sexo feminino na indústria da construção, adaptando modelo de Hurrell e Murphy(113) . Apesar de ser voltado a um gênero específico, assim como para o estresse e não propriamente para o bem-estar, o estudo forneceu uma visão ampla sobre os estressores do trabalho e suas consequências.

Segundo estes autores os estressores são divididos em 3 grandes categorias (que diferem da classificação utilizada no Quadro 3 por ser focado no estresse): (a) demandas do trabalho ou da tarefa (demandas próprias do trabalho, controle e responsabilidades); (b) fatores organizacionais tais como, segurança, assédio, treinamento; (c) e exposições físico químicas. Estes estressores são moderados por aspectos como suporte social e liderança dos supervisores, por exemplo, provocando reações agudas psicológicas (como tensão, raiva e tristeza), fisiológicas (como dor de cabeça, insônias e náuseas), assim como avaliações negativas quanto a satisfação do trabalho (medida utilizada com frequência para Rev. Gestão \& Saúde (Brasília) Vol. 11, n. 01, Jan. 2020. medir o bem-estar do trabalho). Tais circunstâncias levam a doenças como hipertensão, depressão, assim como lesões e acidentes.

É importante frisar que a vinculação conceitual do estresse ao bem-estar é encontrada com frequência na literatura organizacional, assim como diversos outros aspectos, sendo a compreensão específica do construto BET mais escassa(14). Os resultados da presente RSL corroboram com tal perspectiva, apresentando como principal aspecto pesquisado o estresse no trabalho, o qual se relaciona, mas não representa a amplitude do construto do BET.

Ainda assim, a abordagem de Goldenhar et al.(112) ratifica que os principais grupos de aspectos apresentados no Quadro 3 refletem razoavelmente a singularidade dos profissionais da indústria da construção. A propósito, os aspectos apresentados neste quadro trazem alguns resultados já esperados quando se trata do ramo de atividade de construção. A princípio, temáticas dos grupos de segurança, bem como de saúde física, são tratados com frequência na academia. Em publicações voltadas especificamente a engenharia, preponderam tais estudos. A explicação disso pode ser baseada na própria natureza arriscada do trabalho, assim como no histórico de acidentes e lesões que esta indústria apresenta em geral.

Entretanto, os aspectos relacionados aos grupos de medidas de BET, estresse e 
estressores (que representam a maioria pesquisada) e saúde mental são bem disseminados em áreas de saúde, sendo pouco encontrados em eventos e periódicos voltados a engenharia civil. Tal fato, pode gerar um distanciamento entre a teoria e a prática, dificultando o desenvolvimento e a aplicação de melhorias nos canteiros.

\section{Conclusão}

A presente pesquisa teve como objetivo identificar os principais aspectos relacionados ao bem-estar dos profissionais da indústria da construção. Por meio de uma RSL, a qual possui um método rígido, robusto e rastreável, identificaram-se 89 aspectos em 92 publicações acadêmicas. Com características variadas, os principais aspectos foram agrupados metodologicamente por afinidade temática em 6 grupos, o que fornece um melhor entendimento global. Identificaram-se traços comuns a outras pesquisas no que tange a heterogeneidade de conceitos vinculadas ao bem-estar, fato que dificulta pesquisas na área e reforça a necessidade de convergência, apesar da abrangência do tema. Outra conclusão relevante encontra-se na escassez de pesquisas nacionais, bem como da necessidade de um enfoque que vá além de fatores de qualidade de vida em canteiros ou relacionadas a indústria da construção, tais como estresse, bem-estar psicológico e relação trabalho e família, por exemplo. Por fim, recomenda-se, como sugestão para trabalhos futuros:

Rev. Gestão \& Saúde (Brasília) Vol. 11, n. 01, Jan. 2020.
1. Estudos que investiguem e determinem de forma concisa e sistemática os aspectos que proporcionam o BET em trabalhadores da construção. A variedade de aspectos pesquisados representa uma barreira expressiva para tal evolução.

2. Desenvolvimento de protocolos específicos de diagnóstico ou intervenções que possibilitem as entidades mapear e melhorar o BET dos profissionais da construção, possibilitando atuações efetivas neste nicho de profissionais.

3. Pesquisas voltadas aos aspectos positivos do trabalho baseados na psicologia positiva. Tal temática visa o foco de estudos não em pensamentos ruins, mas em aspectos positivos da vida, os quais podem ser espelhados para o ambiente de trabalho. 


\section{Referencias}

1. Ryan RM, Deci EL. On happiness and human potentials: a review of research on hedonic and eudaimonic well-being. Annu Rev Psychol. 2001;52(1):141-66.

2. Baselmans BML, Bartels M. A genetic perspective on the relationship between eudaimonic -and hedonic well-being. Sci Rep [Internet]. 2018;8(1):1-10. Available from: http://dx.doi.org/10.1038/s41598-01832638-1

3. Diener E, Emmons RA, Larsen RJ, Sharon G. The satisfaction with life scale. J Pers Assess. 1985;49(1):71-5.

4. Waterman AS. Two conceptions of happiness: contrasts of personal expressiveness (eudaimonia) and hedonic enjoyment. J Pers Soc Psychol [Internet]. 1993;64(4):678-91. Available from: http://psycnet.apa.org/fulltext/199325585-001.html

5. Diener E. Subjective well-Being. Psychol Bull. 1984;95(3):542-75.

6. Sacks DW, Wolfers J, Stevenson B. The new stylized facts about income and subjective well-being. Emotion. 2012;12(6):1181-7.

7. Adler A, Seligman MEP. Using wellbeing for public policy: Theory, measurement, and recommendations. Int J Wellbeing. 2016;6(1):1-35.

8. Ryff CD. Happiness Is everything, or is it? Explorations on the meaning of psychological well-being. J Pers Soc Psychol [Internet]. 1989;57(6):106981. Available from: http://doi.apa.org/getdoi.cfm?doi=10.1 037/0022-3514.57.6.1069

9. Ryff CD, Keyes CLM. The structure of psychological well-being revisited. J Pers Soc Psychol. 1995;69(4):719-27.

10. Wright TA, Cropanzano R.
Psychological well-Being and job satisfaction as predictors of job performance. J Occup Health Psychol. 2000;5(1):84-94.

11. Sánchez-García JC, Vargas-Morúa G, Hernández-Sánchez BR. Entrepreneurs' well-being: A bibliometric review. Front Psychol. 2018;9:1-19.

12. Salvador-Carulla L, Lucas R, AyusoMateos JL, Miret M. Use of the terms "wellbeing" and "quality of Life" in health sciences: A conceptual framework. Eur J Psychiatry [Internet]. 2014;28(1):50-65. Available from: http://scielo.isciii.es/pdf/ejpen/v28n1/o riginal5.pdf

13. Blanchflower DG, Oswald AJ. Wellbeing over time in Britain and the USA. J Public Econ. 2004;88(7-8):1359-86.

14. Paschoal T, Tamayo A. Construção e validação da escala de bem-estar no trabalho. Avaliação Psicológica [Internet]. 2008;7(1):11-22. Available from: http://pepsic.bvsalud.org

15. Fisher CD. Happiness at work. Int $\mathbf{J}$ Manag Rev. 2010;12:384-412.

16. Dessen MC, Paz M das GT da. Bemestar pessoal nas organizações: $\mathrm{O}$ impacto de configurações de poder e Características de personalidade. Psicol Teor e Pesqui [Internet]. 2010;26(3):549-56. Available from: http://search.proquest.com.ezpprod1.hul.harvard.edu/docview/857120 101 ? accountid $=11311$

17. World Health Organization. Declaration on Workers Health. World Heal Organ. 2006;1-4.

18. Sender G, Fleck D. As organizações e a felicidade no trabalho: Uma perspectiva integrada. Rev Adm Contemp [Internet]. 2017;21(6):764-87. Available from: http://www.scielo.br/scielo.php?script= sci_arttext\&pid=S14156555201700060 


\section{4\&lng=pt\&tlng=pt}

19. Fogaça N. Uma perspectiva multinível da relação entre desempenho, bemestar, justiça e suporte organizacional. [Brasília]: Universidade de Brasília; 2018.

20. Silva CA da, Ferreira MC. Dimensões e indicadores da qualidade de vida e do bem-estar no trabalho. Psicol Teor e Pesqui. 2013;29(3):331-9.

21. Mostert K, Peeters M, Rost I. Workhome interference and the relationship with job characteristics and well-Being: A South African study among employees in the construction industry. Stress Heal. 2011;27(3):e238-51.

22. Siqueira MMM, Orengo V, Peiró JM. Bem-estar no trabalho. In: Novas medidas do comportamento organizacional: ferramentas de diagnóstico e de gestão. Porto Alegre: Artmed; 2014. p. 39-51.

23. Deacon C, Smallwood J, Haupt T. The health and well-being of older construction workers. Int Congr Ser. 2005;1280:172-7.

24. Gibb A, Cook S, Nyateka N, Bust P, Jones W, Finneran A. Wearable simulations for ill-health conditions in construction. Proc Inst Civ Eng - Civ Eng. 2015;168(6):51-6.

25. Filho JBPD, Neto J de PB, Mourão A, Rocha AB da, Luccas AV, Saggin A. Respect for People'S Well-Being: Meditation for Construction Workers. In: 26th Annual Conference of the International Group for Lean Construction. 2018. p. 1160-9.

26. Bowen P, Edwards P, Lingard H, Cattell K. Predictive modeling of workplace stress among construction professionals. J Constr Eng Manag. 2014;140(3):04013055-1:10.

27. Sunindijo RY, Kamardeen I. Work stress Is a threat to gender diversity in Rev. Gestão \& Saúde (Brasília) Vol. 11, n. 01, Jan. 2020. the construction industry. J Constr Eng Manag. 2017;143(10):04017073.

28. Sang K, Dainty A, Ison S. Warning: Working in construction may be harmful to your psychological wellbeing! In: People and Culture in Construction. Taylor \& Francis; 2007. p. 340.

29. Du Plessis K, Cronin D, Corney T, Green E. Australian Blue-Collar Men's Health and Well-Being: : Contextual Issues for Workplace Health Promotion Interventions. Health Promot Pract. 2013;14(5):715-20.

30. Jebelli H, Khalili MM, Hwang S, Lee S. A supervised learning-based construction workers' stress recognition using a wearable electroencephalography (EEG) Device. In: Proceeding of Construction Research Congress 2018 [Internet]. 2018. p. 148-57. Available from: https://ascelibrary.org/doi/pdf/10.1061/ 9780784481301

31. Martin-Halls S. An Olympic effort. Occupational Health [Internet]. 2009 [cited 2018 Dec 5]; Available from: http://link.galegroup.com/apps/doc/A2 68984712/AONE?u=capes\&sid=AON E\&xid=bbf1a6e8.

32. Junior LP, Silva JJR, Júnior BB. O perfil do trabalhador da construção civil no estado de pernambuco como limitante da qualidade do setor. In: XII Encontro Nacional de Tecnologia do Ambiente Construído. Fortaleza; 2008.

33. Neri M. O novo velho trabalhador da construção civil. Revista Conjuntura Econômica. 2011;46-9.

34. Zafar J, Chileshe N. Analysis of health and well-being practices among older construction site-based workers in South Australia. In: Proceeding of the 34th Annual ARCOM Conference. Belfast, UK; 2018. p. 341-50. 
35. Ferraz RRN, Aquino S. Litíase urinária em trabalhadores da construção civil como indicador para a gestão em saúde e melhoria na gestão de pessoas. Cien Saude Colet. 2014;19(12):4759-66.

36. Townsend $\mathrm{K}$, Lingard H, Bradley L, Brown K. Complicated working time arrangements: Construction industry case study. J Constr Eng Manag. 2012;138(3):443-8.

37. Powell A, Galea N, Salignac S, Loosemore M, Chappell L. Masculinity and workplace wellbeing in the Australian construction industry. In: Proceeding of the 34th Annual ARCOM Conference. Belfast, UK; 2018. p. 32130.

38. Kines P, Andersen LPS, Spangenberg S, Mikkelsen KL, Dyreborg J, Zohar D. Improving construction site safety through leader-based verbal safety communication. J Safety Res [Internet]. 2010;41(5):399-406. Available from: http://dx.doi.org/10.1016/j.jsr.2010.06. 005

39. Chih Y-Y, Kiazad K, Zhou L, Capezio A, Li M, Restubog SLD. Investigating employee turnover in the construction industry: A psychological contract perspective. J Constr Eng Manag [Internet]. 2016;142(6):04016006. Available from: http://ascelibrary.org/doi/10.1061/\%28 ASCE\%29CO.1943-7862.0001101

40. Bierman M, Marnewick A, Pretorius JH. Productivity management in the South African civil construction industry - factors affecting construction productivity. J South African Inst Civ Eng [Internet]. 2016;58(3):37-44. Available from: https://ujdigispace.uj.ac.za

41. Konstantinou E. Vision-based construction worker task productivity monitoring. University of Cambridge; 2017.
42. Chih Y, Kiazad K, Cheng D, Capezio A, D. Restubog SL. Does organizational justice matter? Implications for construction workers' organizational commitment. J Manag Eng. 2017;33(2):04016043.

43. Anger WK, Kyler-Yano J, Vaughn K, Wipfli B, Olson R, Blanco M. Total worker health intervention for construction workers alters safety, health, well-being measures. J Occup Environ Med. 2018;60(8):700-9.

44. Lorente L, Salanova M, Martínez IM, Vera M. How personal resources predict work engagement and self-rated performance among construction workers: A social cognitive perspective. Int J Psychol. 2014;49(3):200-7.

45. Hirschle ALT, Gondim SMG. Estresse e bem-estar no trabalho: Uma revisão de literatura. Cien Saude Colet [Internet]. 2018; Available from: http://www.cienciaesaudecoletiva.com. br/artigos/estresse-e-bemestar-notrabalho-uma-revisao-deliteratura/17003

46. Santos GB, Ceballos AG da C de. Bemestar no trabalho: Estudo de revisão. Psicol em Estud. 2013;18(2):247-55.

47. Häggman-Laitila A, Romppanen J. Outcomes of interventions for nurse leaders' well-being at work: A quantitative systematic review. J Adv Nurs. 2018;74(1):34-44.

48. Romppanen J, Häggman-Laitila A. Interventions for nurses' well-being at work: a quantitative systematic review. J Adv Nurs. 2017;73(7):1555-69.

49. Dresch A, Lacerda DP, Júnior JAVA. Design science research: método de pesquisa para avanço da ciência e tecnologia. Design science research: método de pesquisa para avanço da ciência e tecnologia. Porto Alegre: Bookman; 2015. 
50. Abraham S. Employee Retention Requires A Plan. Constr Bull [Internet]. 2007;292(26). Available from: https://search.proquest.com/docview/2 35804154 ? accountid $=26544$

51. Buck MA. Proactive personality and big five traits in supervisors and workgroup members: Effects on safety climate and safety motivation. [Internet]. Portland State University; 2011. Available from: http://search.ebscohost.com/login.aspx ?direct $=$ true $\& \mathrm{db}=$ psyh $\& \mathrm{AN}=2012$ 99060-242\&login. asp\&site $=$ ehost live $\&$ scope $=$ site

52. Choi SD. A study of trade-specific occupational ergonomics considerations in the U.S. construction industry. Work. 2012;42:215-22.

53. Gatti U, Migliaccio G, Bogus SM, Priyadarshini S, Scharrer A. Using Workforce's Physiological Strain Monitoring to Enhance Social Sustainability of Construction. J Archit Eng. 2013;19(3):179-85.

54. Hinze J, Godfrey R, Sullivan J. Integration of Construction Worker Safety and Health in Assessment of Sustainable Construction. J Constr Eng Manag. 2013;139(6):594-600.

55. Albert A, Hallowell MR, Kleiner B, Chen A, Golparvar-Fard M. Enhancing Construction Hazard Recognition with High-Fidelity Augmented Virtuality. J Constr Eng Manag [Internet]. 2014;140(7):04014024. Available from: http://ascelibrary.org/doi/10.1061/\%28 ASCE\%29CO.1943-7862.0000860

56. Bodner $\mathrm{T}$, Kraner $\mathrm{M}$, Bradford $\mathrm{B}$, Hammer L, Truxillo DM. Safety, Health, and Well-Being of Municipal Utility and Construction Workers. J Occup Environ Med. 2014;56(7):7718.

57. Costa ACB. The effects of organizational justice and exercise on the relationship between job stressors and employee health. Portland State University; 2014.

58. Gatti UC, Schneider S, Migliaccio GC. Physiological condition monitoring of construction workers. Autom Constr [Internet]. 2014;44:227-33. Available from:

http://dx.doi.org/10.1016/j.autcon.2014 .04 .013

59. Kaufman BR, Cigularov KP, Chen P, Hoffmeister K, Gibbons AM, Johnson SK. Interactive effects of leader justice and support for safety on safety performance. J Organ Eff People Perform. 2014;1(3):43-69.

60. Arias OE, Caban-Martinez AJ, Umukoro PE, Okechukwu CA, Dennerlein JT. Physical activity levels at work and outside of work among commercial construction workers. J Occup Environ Med. 2015;57(1):73-8.

61. Dailey S. Firms balance profit and employee well- being. ENR: Engineering News-Record. 2015;

62. Lee W, Lin KY, Seto E, Migliaccio GC. Wearable sensors for monitoring onduty and off-duty worker physiological status and activities in construction. Autom Constr [Internet]. 2017;83(August 2016):341-53. Available from: http://dx.doi.org/10.1016/j.autcon.2017 .06 .012

63. Gatti UC, Lin K-Y, Caldera C, Chiang R. Exploring the Relationship between Chronic Sleep Deprivation and Safety on Construction Sites. Constr Res Congr 2014. 2014;1792-800.

64. Curtis HM, Meischke H, Stover B, Simcox NJ, Seixas NS. Gendered safety and health risks in the construction trades. Ann Work Expo Heal. 2018;62(4):404-15.

65. Jebelli H, Hwang S, Lee SH. EEG- 
based workers' stress recognition at construction sites. Autom Constr [Internet]. 2018;93:315-24. Available from:

https://doi.org/10.1016/j.autcon.2018.0 5.027

66. Peters SE, Grant MP, Rodgers J, Manjourides J, Okechukwu CA, Dennerlein JT. A cluster randomized controlled trial of a total worker health (B) intervention on commercial construction sites. Int $\mathrm{J}$ Environ Res Public Health. 2018;15(2353):1-20.

67. Thomas NA. Project manager trait emotional intelligence and project success [Internet]. Walden University; 2018. Available from: http://search.ebscohost.com/login.aspx ?direct=true $\&$ AuthType $=i p$, shib,cpid $\&$ custid $=\mathrm{s} 6264444 \& \mathrm{db}=$ psyh $\& A N=2018$ $-11221-108 \&$ site $=$ ehost -

live $\&$ scope $=$ site

68. Lauren Tosti. Creating a culture of safety on construction sites. Occup Heal Saf [Internet]. 2018; (july 2018). Available from: https://ohsonline.com/articles/2018/07/ 01/creating-a-culture-of-safety.aspx

69. Lingard H, Francis V. Does workfamily conflict mediate the relationship between job schedule demands and burnout in male construction professionals and managers? Constr Manag Econ. 2005;23(7):733-45.

70. Love PED, Edwards DJ, Irani Z. Work Stress, Support, and Mental Health in Construction. J Constr Eng Manag. 2010;136(6):650-8.

71. Turner M, Lingard H. Demands and resources of workers in the Australian construction industry: Identification and exploration using $\mathrm{Q}$ methodology. In: Procs 27th Annual ARCOM Conference [Internet]. Bristol, UK; 2011. p. 361-70. Available from: http://www.scopus.com/inward/record. url?eid=2-s2.0-

Rev. Gestão \& Saúde (Brasília) Vol. 11, n. 01, Jan. 2020.
84861034854\&partnerID=tZOtx3y1

72. Milner A, Niven H, Lamontagne A. Suicide by occupational skill level in the Australian construction industry: Data from 2001 to 2010. Aust N Z J Public Health. 2014;38(3):281-5.

73. Zacher H, Jimmieson NL, Bordia P. Time pressure and coworker support mediate the curvilinear relationship between age and occupational wellbeing. J Occup Health Psychol. 2014;19(4):462-75.

74. Milner A, Witt K, Burnside L, Wilson C, Lamontagne AD. Contact \& connect - an intervention to reduce depression stigma and symptoms in construction workers: protocol for a randomised controlled trial. BMC Public Health [Internet]. 2015;15(1):1-6. Available from: http://dx.doi.org/10.1186/s12889015-2394-x

75. Turner M, Lingard H. Work-life fit: identification of demand and resource typologies within a systems framework. Constr Manag Econ. 2016;34(6):37792.

76. Turner M, Mariani A. Managing the work-family interface: experience of construction project managers. Int $\mathbf{J}$ Manag Proj Bus. 2016;9(2):243-58.

77. Loudoun R, Townsend K. Implementing health promotion programs in the Australian construction industry: Levers and agents for change. Eng Constr Archit Manag. 2017;24(2):260-74.

78. Pidd K, Duraisingam V, Roche A, Trifonoff A. Young construction workers: substance use, mental health, and workplace psychosocial factors. Adv Dual Diagn. 2017;10(4):155-68.

79. King TL, Gullestrup J, Batterham PJ, Kelly B, Lockwood C, Lingard H, et al. Shifting beliefs about suicide: Pre-post evaluation of the effectiveness of a 
program for workers in the construction industry. Int $\mathrm{J}$ Environ Res Public Health. 2018;15(2106):1-13.

80. Kurtzer D, Blackmore N, Farrugia N, Chileshe N. Productivity enablers and inhibiting health and wellbeing practices of South Australian construction site-based workers: a qualitative study. Int $\mathrm{J}$ Constr Manag [Internet]. 2018;1-18. Available from: https://doi.org/10.1080/15623599.2018 .1496538

81. Langdon RR, Sawang S. Construction Workers' Well-Being: What Leads to Depression, Anxiety, and Stress? J Constr Eng Manag [Internet]. 2018;144(2):15p. Available from: http://ascelibrary.org/doi/10.1061/\%28 ASCE\%29CO.1943-7862.0001406

82. Sutherland V, Davidson MJ. Stress among construction site managers - A preliminary study. Stress Med. 1989;5:221-35.

83. Love PED, Edwards DJ. Taking the pulse of UK construction project managers' health: Influence of job demands, job control and social support on psychological wellbeing. Eng Constr Archit Manag. 2005;12(1):88-101.

84. Sang KJC, Dainty ARJ, Ison SG. Gender: A risk factor for occupational stress in the architectural profession? Constr Manag Econ. 2007;25(12):1305-17.

85. Sang KJC, Ison SG, Dainty ARJ. The job satisfaction of UK architects and relationships with work-life balance and turnover intentions. Eng Constr Archit Manag. 2009;16(3):288-300.

86. Tyers C, Hicks B, Baxter K, Gilbert M. Learning from the occupational health provision on the Olympic build. Policy Pract Heal Saf. 2012;10(2):103-23.

87. Sherratt F. We will force you to be well: Positive liberty, power and the health and wellbeing of construction workers. In: 30th Annual ARCOM Conference. Portsmouth, UK; 2014. p. 341-50.

88. Aboagye-Nimo E, Raiden A, King A, Tietze S. Using tacit knowledge in training and accident prevention. Manag Procure Law. 2015; 168(MP5).

89. Eaves SJ, Gyia DE, Gibb GF. Facilitating healthy ageing in construction: stakeholder views. Procedia Manuf. 2015;0-8.

90. Eaves S, Gyi DE, Gibb AGFF. Building healthy construction workers: Their views on health, wellbeing and better workplace design. Appl Ergon [Internet]. 2016;54:10-8. Available from:

http://dx.doi.org/10.1016/j.apergo.2015 .11 .004

91. Valero E, Sivanathan A, Bosché F, Abdel-Wahab M. Musculoskeletal disorders in construction: A review and a novel system for activity tracking with body area network. Appl Ergon. 2016;54:120-30.

92. Cole M. Construction employers are increasing efforts to tackle often unseen mental health issues which affect the industry even more? New Civil Engineer. 2017;29-33.

93. Cole M. As construction fatalites fall, the danger is complacency. so how can the industry engage with its employees to drive accident rates down even more? New Civil Engineer. 2017;34-6.

94. Smallwood JJ, Haupt TC. Construction ergonomics: perspectives of female and male production workers. In: 25th Annual ARCOM Conference. 2009. p. 1263-71.

95. Bowen P, Edwards P, Lingard H, Cattell K. Occupational stress and job demand, control and support factors among construction project consultants. Int J Proj Manag [Internet]. 
2014;32(7):1273-84. Available from: http://dx.doi.org/10.1016/j.ijproman.20 14.01.008

96. Bowen P, Govender R, Edwards P, Cattell K, Street A. Factors Determining South African Construction Workers' Prejudice towards and Discrimination against HIV+ Persons. J Constr Eng Manag [Internet]. 2015;141(7):04015014. Available from:

http://ascelibrary.org/doi/10.1061/\%28 ASCE\%29CO.1943-7862.0000995

97. Bernstein C, Trimm L. The impact of workplace bullying on individual wellbeing: The moderating role of coping. SA J Hum Resour Manag. 2016;15:1-12.

98. Coetzer MF, Bussin MHR, Geldenhuys M. Servant leadership and work-related well-being in a construction company. SA J Ind Psychol. 2017;43:1-10.

99. Obiozo RN, Smallwood J. Ergonomic realities of a Biophilic Construction Site Model. Ergon SA. 2017;29(2):27-40.

100. Meliá JL, Becerril M. Psychosocial sources of stress and burnout in the construction sector: A structural equation model. Psicothema. 2007;19(4):679-86.

101. Fernández MD, Quintana S, Chavarría $\mathrm{N}$, Ballesteros JA. Noise exposure of workers of the construction sector. Appl Acoust [Internet]. 2009;70(5):753-60. Available from: http://dx.doi.org/10.1016/j.apacoust.20 08.07.014

102. Navarro-astor E. Job Perception of Spanish Qualified Construction Professionals. 2010;(September):38190.

103. López-Araújo B, Segovia AO. El papel del modelo Demandas-Control-Apoyo en la salud de trabajadores de la construcción. [The effects of the
Demand-Support-Control model on construction workers' health.]. Psicothema. 2011;23(1):119-25.

104. Chan APCC, Yam MCHH, Chung JWYY, Yi W. Developing a heat stress model for construction workers. J Facil Manag. 2012;10(1):59-74.

105. Yi W, Chan A. Health profile of construction workers in Hong Kong. Int J Environ Res Public Health. 2016;13(12).

106. Yi W, Chan APC, Wang $\mathrm{X}$ WJ. Development of an early-warning system for site work in hot and humid environments: a case study. Autom Constr. 2016;62:101-13.

107. Kong L, Li H, Yu Y, Luo H, Skitmore M, Antwi-Afari MF. Quantifying the physical intensity of construction workers, a mechanical energy approach. Adv Eng Informatics. 2018;38(May):404-19.

108. Yuan J, Yi W, Miao M, Zhang L. Evaluating the Impacts of Health, Social Network and Capital on Craft Efficiency and Productivity: A Case Study of ConstructionWorkers in China. Int J Environ Res Public Health. 2018;15(345):1-25.

109. McCabe B, Loughlin C, Munteanu R, Tucker S, Lam A. Individual safety and health outcomes in the construction industry. Can J Civ Eng. 2008;35(12):1455-67.

110. Alwasel A, Elrayes K, Abdel-Rahman E, Haas C. A human body posture sensor for monitoring and diagnosing MSD risk factors. In: ISARC Proceedings of the International Symposium on Automation and Robotics in Construction. 2013. p. 1.

111. Chen Y, McCabe B, Hyatt D. Impact of individual resilience and safety climate on safety performance and psychological stress of construction 
workers: A case study of the Ontario construction industry. J Safety Res. 2017;61:167-76.

112. Goldenhar LM, Swanson NG, Hurrell J, Ruder A, Deddens J. Stressors and adverse outcomes for female construction workers job stressors. J Occup Health Psychol. 1998;3(1):1932.

113. Hurrell J, Murphy LR. Psychological job stress. Environ Occup Heal. 1992;675-85.

\section{Participação dos autores:}

Moreira FS é o autor principal do texto, o qual foi derivado de parte de sua tese de doutorado. Nesta condição, trabalhou em todas as fases do artigo.

Maués LMF trabalhou na orientação geral do processo de revisão sistemática da literatura, indicando também a abordagem teórica adequada voltado a indústria da construção.

Freitas ACA trabalhou na seleção e tratamento dos artigos para a revisão sistemática, bem como no registro no banco de dados de revisões PROSPERO.

Farias Júnior CA trabalhou na revisão final e formatação do trabalho.

Recebido: 12.09.2019

Revisado: 02.01.2019

Aprovado: 16.01.2020 